Review

\title{
Health consequences of electric lighting practices in the modern world: A report on the National Toxicology Program's workshop on shift work at night, artificial light at night, and circadian disruption
}

Ruth M. Lunn a , David E. Blask ${ }^{\mathrm{b}}$, Andrew N. Coogan ${ }^{\mathrm{c}}$, Mariana G. Figueiro ${ }^{\mathrm{d}}$, Michael R. Gorman ${ }^{\mathrm{e}}$, Janet E. Hall ${ }^{\mathrm{f}}$, Johnni Hansen ${ }^{\mathrm{g}}$, Randy J. Nelson ${ }^{\mathrm{h}}$, Satchidananda Panda ${ }^{\mathrm{i}}$, Michael H. Smolensky ${ }^{\mathrm{j}, \mathrm{k}}$, Richard G. Stevens ${ }^{1}$, Fred W. Turek ${ }^{\mathrm{m}}$, Roel Vermeulen ${ }^{\mathrm{n}}$, Tania Carreón ${ }^{\mathrm{o}}$, Claire C. Caruso ${ }^{\circ}$, Christina C. Lawson ${ }^{\circ}$, Kristina A. Thayer ${ }^{\mathrm{p}}$, Michael J. Twery ${ }^{\mathrm{q}}$, Andrew D. Ewens ${ }^{\mathrm{r}}$, Sanford C. Garner ${ }^{\mathrm{r}}$, Pamela J. Schwingl ${ }^{\mathrm{r}}$, Windy A. Boyd ${ }^{\mathrm{p}, *}$

a Office of the Report on Carcinogens, Division of the National Toxicology Program, National Institute of Environmental Health Sciences (NIEHS), Research Triangle Park, NC, United States

${ }^{b}$ Department of Structural and Cellular Biology, Laboratory of Chrono-Neuroendocrine Oncology, Tulane University School of Medicine, New Orleans, LA, United States

c Maynooth University Department of Psychology, National University of Ireland, Maynooth, County Kildare, Ireland

d Light and Health Program, Lighting Research Center, Rensselaer Polytechnic Institute, Troy, NY, United States

e Department of Psychology and Center for Circadian Biology, University of California, San Diego, CA, United States

${ }^{\mathrm{f}}$ Division of Intramural Research, National Institute of Environmental Health Sciences, Research Triangle Park, NC, United States

g Danish Cancer Society Research Centre, Copenhagen, Denmark

${ }^{\mathrm{h}}$ Department of Neuroscience, Neuroscience Research Institute, The Ohio State University Wexner Medical Center, Columbus, OH, United States

${ }^{i}$ Salk Institute for Biological Studies, La Jolla, CA, United States

j Department of Biomedical Engineering, The University of Texas at Austin, Austin, TX, United States

${ }^{\mathrm{k}}$ Sleep Medicine, The University of Texas-Houston McGovern School of Medicine, Houston, TX, United States

${ }^{1}$ School of Medicine, University of Connecticut, Farmington, CT, United States

m Center for Sleep \& Circadian Biology, Northwestern University, Evanston, IL, United States

${ }^{n}$ Division of Environmental Epidemiology, Institute for Risk Assessment Sciences (IRAS), Utrecht University, Utrecht, The Netherlands

${ }^{\circ}$ National Institute for Occupational Safety and Health (NIOSH), Centers for Disease Control and Prevention, Cincinnati, OH, United States

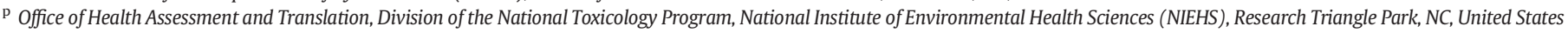

q National Center on Sleep Disorders Research, Division of Lung Diseases, National Heart, Lung, and Blood Institute (NHLBI), Bethesda, MD, United States

${ }^{\mathrm{r}}$ Contractor in support of the NIEHS Report on Carcinogens, Integrated Laboratory Systems (ILS), Durham, NC, United States

\section{H I G H L I G H T S}

- Patterns of light exposure during day and night have direct effects on circadian biology and health.

- Modern electric lighting practices also enable 24/7 activities and may lead to circadian disruption.

- Work at night is a complex exposure scenario, including, but not limited to, light at night.

- Some light and work at night are essential to society, making identification of interventions challenging.

- Better measures of exposure and early disease may help identify interventions to protect public health.
GRAPHICAL A B STRACT
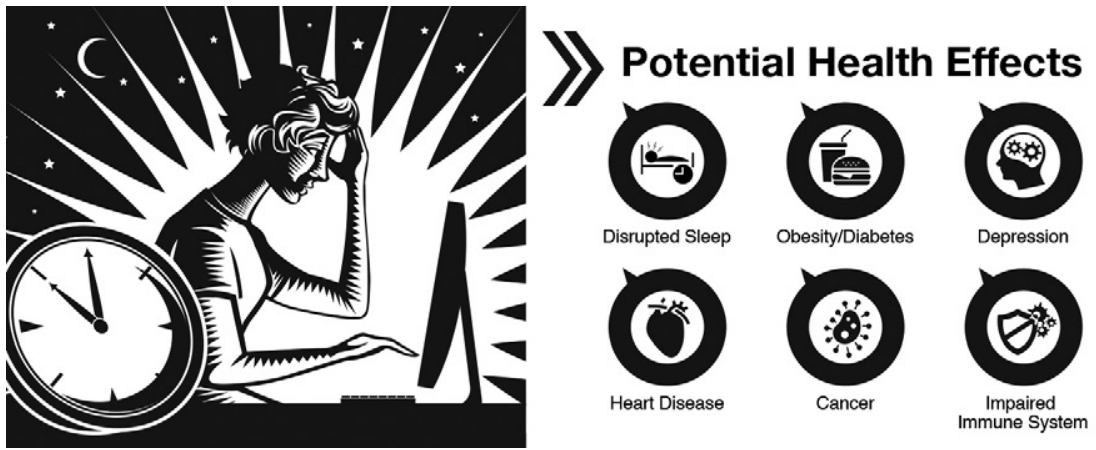

\footnotetext{
* Corresponding author at: PO Box 12233, MD K2-04, RTP, NC 27709, United States.

E-mail address: boydw@niehs.nih.gov (W.A. Boyd).
} 


\section{A R T I C L E I N F O}

\section{Article history:}

Received 8 May 2017

Received in revised form 7 July 2017

Accepted 7 July 2017

Available online 27 July 2017

Editor: Jay Gan

\section{Keywords:}

Artificial light at night (ALAN)

Light at night (LAN)

Shift work

Circadian disruption

\begin{abstract}
A B S T R A C T
The invention of electric light has facilitated a society in which people work, sleep, eat, and play at all hours of the 24-hour day. Although electric light clearly has benefited humankind, exposures to electric light, especially light at night (LAN), may disrupt sleep and biological processes controlled by endogenous circadian clocks, potentially resulting in adverse health outcomes. Many of the studies evaluating adverse health effects have been conducted among night- and rotating-shift workers, because this scenario gives rise to significant exposure to LAN. Because of the complexity of this topic, the National Toxicology Program convened an expert panel at a public workshop entitled "Shift Work at Night, Artificial Light at Night, and Circadian Disruption" to obtain input on conducting literature-based health hazard assessments and to identify data gaps and research needs. The Panel suggested describing light both as a direct effector of endogenous circadian clocks and rhythms and as an enabler of additional activities or behaviors that may lead to circadian disruption, such as night-shift work and atypical and inconsistent sleep-wake patterns that can lead to social jet lag. Future studies should more comprehensively characterize and measure the relevant light-related exposures and link these exposures to both time-independent biomarkers of circadian disruption and biomarkers of adverse health outcomes. This information should lead to improvements in human epidemiological and animal or in vitro models, more rigorous health hazard assessments, and intervention strategies to minimize the occurrence of adverse health outcomes due to these exposures.
\end{abstract}

Published by Elsevier B.V.

\section{Contents}

1. Introduction . . . . . . . . . . . . . . . . . . . . . . . . . . . . . . . . . . . . . . . . . . . . 1074

2. Methods: workshop format . . . . . . . . . . . . . . . . . . . . . . . . . . . . . . . . . 1075

3. Results and discussion . . . . . . . . . . . . . . . . . . . . . . . . . . . . . . . . . . . . . . . . . . . . . 1075

3.1. Light as effector and enabler . . . . . . . . . . . . . . . . . . . . . . . . . . . . . . . . . . . 1075

3.2. Light as an effector . . . . . . . . . . . . . . . . . . . . . . . . . . . . . . . . . . . . . . . . . . . . . . . 1075

3.3. Light as an enabler . . . . . . . . . . . . . . . . . . . . . . . . . . . . . . . . . . . . . . 1075

3.4. Shift work: light as both effector and enabler . . . . . . . . . . . . . . . . . . . . . . . . . . . . . . . . . . . . . . 1076

3.5. Circadian disruption and adverse health effects . . . . . . . . . . . . . . . . . . . . . . . . . . . . . . . . . . . . . . . . . . 1077

3.5.1. Advancing the science for understanding potential health outcomes. . . . . . . . . . . . . . . . . . . . . . . . . 1078

3.6. Characterization of light in human studies . . . . . . . . . . . . . . . . . . . . . . . . . . . . . . . . . . . . 1078

3.7. Characterization of exposures in studies of shift workers . . . . . . . . . . . . . . . . . . . . . . . . . . . . . . . . . 1078

3.8. Animal models of LAN and shift work. . . . . . . . . . . . . . . . . . . . . . . . . . . . . . . . . . . . . . . . . . . . 1079

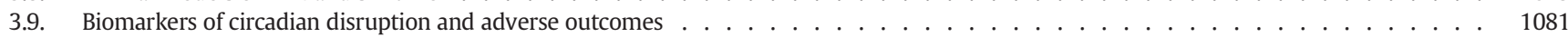

3.9.1. Protecting public health. . . . . . . . . . . . . . . . . . . . . . . . . . . . . . . . . . . 1081

4. Conclusions. . . . . . . . . . . . . . . . . . . . . . . . . . . . . . . . . . . 1082

Funding . . . . . . . . . . . . . . . . . . . . . . . . . . . . . . . . . . . . . . . . . . . . 1083

Disclaimer. . . . . . . . . . . . . . . . . . . . . . . . . . . . . . . . . . . . 1083

Acknowledgments . . . . . . . . . . . . . . . . . . . . . . . . . . . . . . . . . . . . . . . 1083

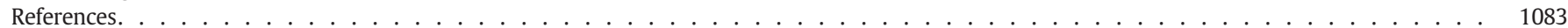

\section{Introduction}

The widespread adoption of electric light, only about 130 years ago, has facilitated a 24-hour/7-day society in which people work, sleep, eat, play, and expect goods and services at any time during the day or night. In this situation, people are exposed to electric light through lifestyle choices (e.g., use of electronic devices at night), the locations of their residences (e.g., urban light pollution), and employment during the night shift or as a shift worker (i.e., a worker whose schedule switches among morning, day, evening, and night). The United Nations proclaimed 2015 the International Year of Light and Light-Based Technologies in recognition of how light "has revolutionized medicine, opened up international communication via the Internet, and continues to be central to linking cultural, economic, and political aspects of the global economy" (http://www.light2015.org/Home/About.html). Although electric light has clearly benefited humankind, exposures to ill-timed, unnatural electric light, such as light at night (LAN) or differences in the quantity and spectrum of electric light from that provided by daylight exposures, may disrupt sleep and biological processes controlled by endogenous circadian clocks, potentially resulting in adverse health outcomes.

Because of these potential health concerns, LAN was nominated to the National Toxicology Program (NTP) to be the subject of literature- based health-hazard assessments on cancer and non-cancer outcomes. The NTP's Office of the Report on Carcinogens (ORoC) is assessing cancer outcomes for potential listing in the RoC, a U.S. congressionally mandated, science-based document that identifies cancer hazards. The Office of Health Assessment and Translation (OHAT) was asked to consider evaluating non-cancer (e.g., cardiovascular, metabolic, reproductive, gastrointestinal, immunological, neurological, and psychiatric) outcomes. Both ORoC and OHAT evaluations typically integrate evidence from human, experimental animal, and mechanistic studies. One of the rationales for nominating LAN to the RoC was the International Agency for Research on Cancer (IARC) working group 2007 conclusion that "shift work involving circadian disruption is probably carcinogenic to humans" (IARC, 2010). Therefore, the NTP defined the nomination to include circadian disruption and shift work at night, in addition to LAN. Those nominating this topic emphasized that they were interested in a broader scope than shift work, because of concerns about effects from exposure to LAN in the sleeping area (from indoor and outdoor lights) and outfitting of institutions with light sources rich in short-wavelength content, such as some types of light-emitting diodes, and based on the opinion that carcinogenic effects observed among shift workers were potentially due to LAN.

Because of the complexity and breadth of the topic, the NTP convened a public workshop and webcast entitled "Shift Work at Night, 
Artificial Light at Night, and Circadian Disruption" on March 10-11, 2016, to help obtain input on the approach for the NTP literature-analysis activities and to identify data gaps and research needs in the field. (The Panel suggested changing the terminology used by the NTP to describe the exposure to light from "artificial light at night" [ALAN] to "electric lighting practices.")

This workshop report captures the major discussion points of the Panel. The first part introduces the concepts of electric light as effector and enabler and of shift work as a complex exposure scenario, and it provides background information on circadian disruption and links to potential adverse health effects. The second part discusses key issues related to advancing the science, which will help inform NTP literaturebased evaluations and identify data gaps and research needs. This section is followed by a brief discussion of interventions to protect public health. Finally, post-workshop activities and meeting outcomes are discussed. It is important to note that the workshop did not require expert consensus and is not intended to provide formal recommendations or guidelines.

\section{Methods: workshop format}

To meet the workshop goals, a cross-disciplinary workshop panel was selected by the NTP with experts in (1) the key exposures, such as light, shift work, and sleep, (2) health outcomes, such as cancer and reproductive, neurological, and behavioral outcomes, and (3) other pertinent disciplines, such as circadian biology, exposure assessment, epidemiology, clinical research, toxicology, and mechanisms of disease.

The workshop was organized into six sessions, which reflected a progression of knowledge within each session informing subsequent sessions. The workshop began with a description of circadian disruption, followed by cross-disciplinary dialogues on exposure scenarios of LAN, shift work, and additional overlapping exposures related to circadian disruption, such as sleep and timing of meals. It concluded with sessions on strategies to synthesize information across the various exposure scenarios and identify data gaps and research needs. The first three sessions included brief presentations by the experts on the state of the science that defined the topic and provided an overview of the research in humans or experimental animals. All sessions included a moderator-led period with specific questions related to interpretation of the science that had been developed by the NTP prior to the workshop. In addition to discussion questions, to provide a brief introduction to the topics and facilitate workshop discussions, the NTP prepared abstracts and appendices, including tables and key references for each session, organized by health outcomes within the broad categories of cancer and non-cancer outcomes. The workshop was webcast and provided opportunity for public comment by those attending the meeting in person. More information and links to videos of the webcast are available on the workshop website at http://ntp.niehs.nih.gov/go/workshop_ALAN.

\section{Results and discussion}

\subsection{Light as effector and enabler}

One of the key suggestions from the Panel was to describe light both as a direct effector on circadian rhythms and as an enabler of additional activities that may lead to circadian disruption, including night-shift work and other atypical and inconsistent sleep-wake patterns that can lead to social jet lag. It is important to note that the NTP workshop focused on effects of light and shift work on the circadian domain of the biological time structure. Circadian disruption was defined by the panel as internally or externally induced disorganization, including, but not limited to, misalignment of time structure in living systems, potentially leading to adverse health outcomes (for more discussion, see Smolensky et al. (2016)). In addition to circadian rhythms, biological processes are organized as (1) ultradian rhythms, with periods shorter than a day, typically in the 90-minute to 6-hour range (e.g., the duration of different sleep stages), (2) infradian rhythms, with cycles longer than $28 \mathrm{~h}$ (e.g., the menstrual cycle), and (3) seasonal rhythms (e.g., annual variation in endocrine parameters, metabolism, and appetite that are entrained by variation in day length) (Blask et al., 2009). The different biological rhythm domains are likely interconnected so as to contribute to the overall functional and adaptive capacity of life.

\subsection{Light as an effector}

Light is essential to life and critical for the regulation of circadian rhythms. These 24-hour rhythms of daily physiological processes are coordinated by internal biological clocks that are regulated at the molecular level by specific "clock genes." These genes and their protein products oscillate on a self-sustained, near-24-hour basis, but are synchronized to a 24-hour period by input from external and internal time cues, and they influence a suite of circadian rhythms, such as sleep-wake cycles and cycles of body temperature, blood pressure, and metabolism. For most of human existence, light came in the form of direct and diffuse sunlight during the day and nearly complete darkness at night, with the exception of light from the moon, stars, and firelight. In modern society, unnatural exposures to light, including irregular light-dark patterns characterized by insufficient light exposure during the day, too much LAN, or a combination of both, are ubiquitous.

The suprachiasmatic nuclei (SCN) of the hypothalamus sit at the top of a hierarchy of endogenous biological clocks that regulate circadian rhythms in physiology and behavior. The so-called master circadian clock is synchronized to the 24-hour "day" by exposure to the ambient light-dark cycle, primarily by light transmitted via retinal photoreceptors (both image-forming and non-image-forming photoreceptors in the outer and inner retina) to the SCN. Signals are then relayed from the master clock to a multitude of peripheral clocks located in almost every cell of the body, including the melatonin-secreting pineal gland. This orchestration of organismal rhythmicity by the SCN occurs through diverse mechanisms that include neural, hormonal, metabolic, and thermal networks to synchronize (or "entrain") all circadian rhythms and ensure appropriate alignment between internal and external time (Fig. 1). One important biochemical signal is the hormone melatonin. In response to signals from the SCN conveyed via the sympathetic chain, melatonin is primarily produced in the pineal gland of the brain and secreted into the general circulation, where it is made available to peripheral tissues. Melatonin is influenced by the SCN and is also a chronobiotic feedback moderator of the oscillatory activity of the SCN, whose neurons express melatonin receptors and are neurophysiologically responsive to melatonin (Fig. 1) (Kandalepas et al., 2016). Nocturnal light exposures directly suppress pineal production of melatonin, a robust marker of circadian rhythmicity. In the absence of LAN, levels of melatonin in blood, cerebral spinal fluid, and saliva are normally low during the day and high during the darkness of night. The timing, duration, quantity, and spectrum of light exposures throughout the day and evening influence the duration, amplitude, and total quantity of melatonin production during the night. In seasonal animals, duration of the melatonin signal is a physiological proxy for day length (short signal for long summer days and long signal for short winter days). Light, especially of certain wavelength characteristics, will acutely curtail nocturnal production.

\subsection{Light as an enabler}

Electricity has allowed for major advances in modern-day society and can be considered an enabler of circadian disruption. This is principally because electric LAN allows people to work, eat, and enjoy leisure time during the night, with concomitant indirect effects on sleep timing (e.g., night-shift workers who sleep during the day). As a result, significant and rapid alterations have occurred in the night-time environment, both outdoors and inside the home and workplace. Satellite images of Earth at night have revealed how ubiquitous exposures to LAN have 


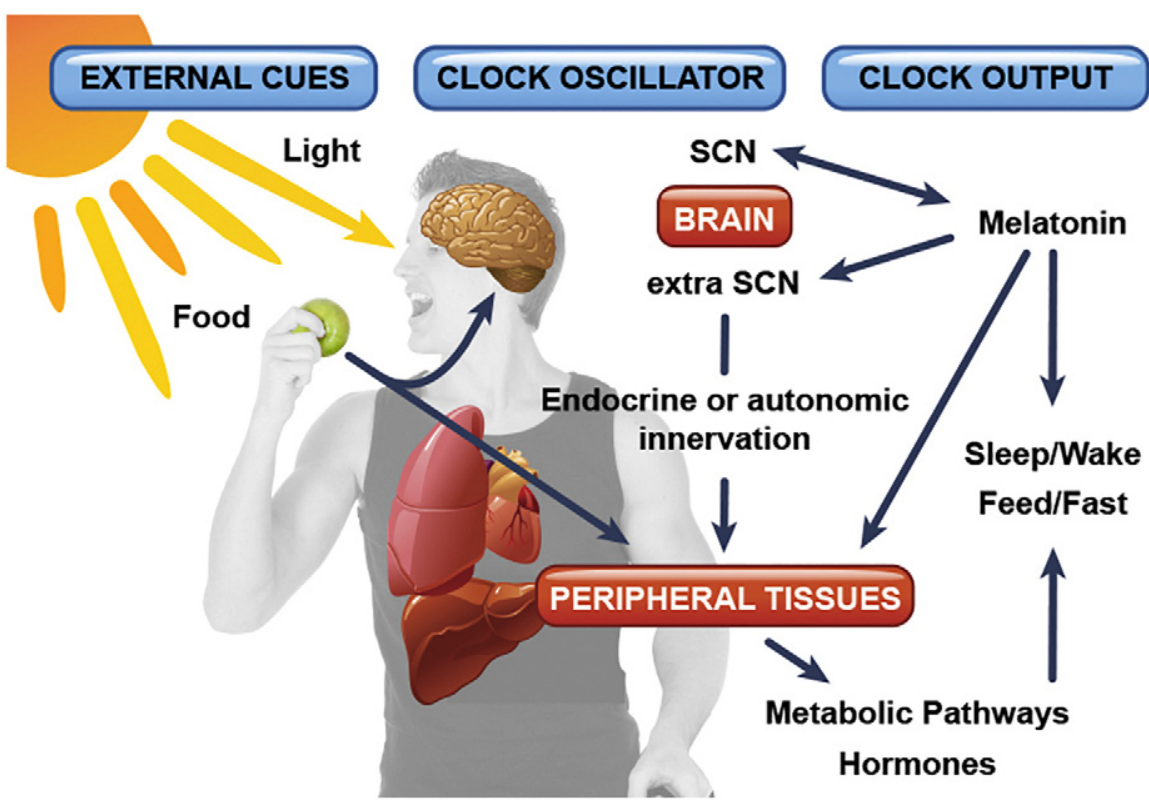

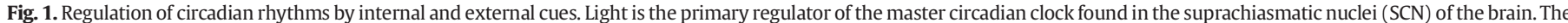

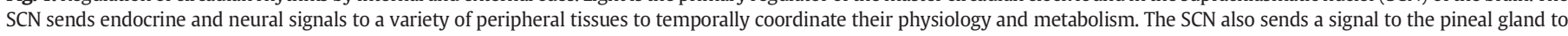

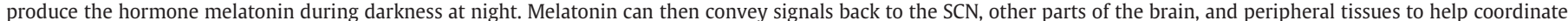
physiological functions and behaviors to approximate 24-hour days.

become over the past several decades in major cities and surrounding areas, as LAN now covers 80\% of the world (Falchi et al., 2016) (Fig. 2). Perhaps not surprisingly, self-reported sleep duration among adults in those areas with the greatest levels of LAN also show a lower prevalence of healthy sleep duration, defined as 7 or more hours of sleep within a 24-hour period. A study in the United States among non-institutionalized people aged 18 years or older found that living in areas with greater outdoor nighttime light was significantly associated with delayed bedtime and wake up time, shorter sleep duration, increased daytime sleepiness, and greater dissatisfaction with sleep quantity and quality (Ohayon and Milesi, 2016). Meanwhile, increasing numbers of people, including children and adolescents, who may be developmentally vulnerable to sleep deprivation, circadian disruption, and downstream effects of such disruption, are using electronic devices, such as televisions, computers, tablets, and smartphones, in the evenings and nights, often shortly before commencing sleep. Some studies have found that adolescents are more sensitive than adults to circadian disruption by evening light, as measured by acute melatonin suppression (Crowley and Carskadon, 2010; Figueiro and Overington, 2015).

\subsection{Shift work: light as both effector and enabler}

In addition to traditional jobs necessarily carried out both day and night, such as health care, security, and emergency response, economic globalization has increased the need for work environments that allow businesses and organizations to operate longer than the hours of an individual worker and thus produce goods and services on a 24/7 basis. Shift work typically involves working outside the standard daytime hours ( 7 a.m. to 6 p.m.) - such as at night, during evenings, or starting before 6 a.m. - and shifts can be permanent, slowly alternating, rapidly switching from day to night (forward rotating) or night to day (backward rotating), or without any particular pattern. Night-shift work is a common occupational exposure; approximately $15 \%$ to $18 \%$ of all

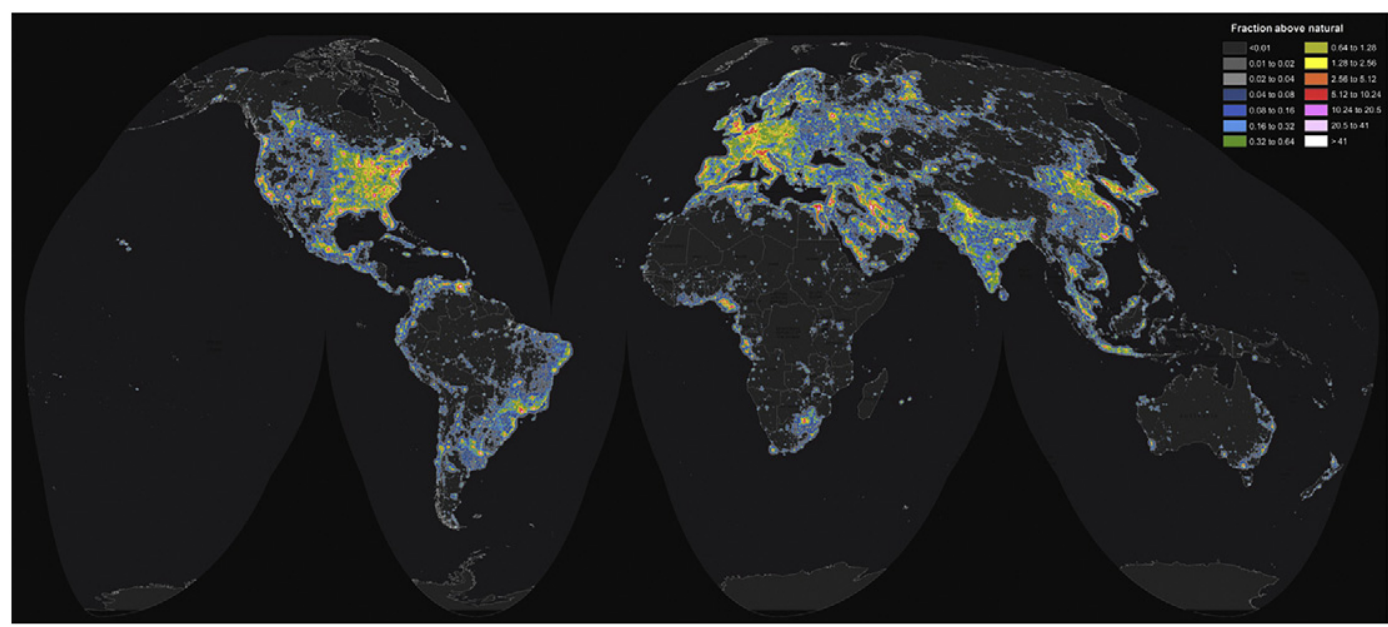

Fig. 2. World map of artificial brightness as a ratio to the natural sky brightness (Falchi et al., 2016). 
workers in Europe and the United States work a variety of night-shift schedules (Straif et al., 2007).

In a typical night-shift work scenario, exposure to LAN that is effective at suppressing melatonin or disrupting the circadian system can occur (often for the entire nighttime period over decades of employment), which may result in periodic or chronic circadian disruption. Field studies reporting on actual LAN exposure are not widely available, but a recent review suggests that it can range from 50 to 100 photopic lux at the eye, with some exposures exceeding $200 \mathrm{~lx}$ at the eye (Hunter and Figueiro, 2017). Studies of shift workers were originally conducted to test the hypothesis that increasing exposure to LAN is responsible, in part, for the increasing incidence of breast cancer in highincome countries (see "Circadian Disruption and Adverse Health Effects," below). IARC concluded in 2007 that shift work involving circadian disruption is probably carcinogenic to humans (Group 2A), based on limited evidence of carcinogenicity for breast cancer from eight human studies, in addition to sufficient evidence from studies in experimental animals. Other studies of shift workers have evaluated risk for other types of cancer outcomes (most notably prostate and colorectal cancer and to a lesser degree other hormonal cancers, including ovarian and endometrial cancers) and non-cancer outcomes (especially cardiovascular disease, diabetes, obesity, mental health and other neurological disorders, and disorders of the endocrine and reproductive systems) (see webinar meeting materials available at http://ntp.niehs.nih.gov/ go/workshop_ALAN).

Although shift work may represent an extreme type of LAN exposure, it is not an exact surrogate for LAN, because it is a complex exposure scenario. In addition to direct effects due to LAN and altered exposure to daylight, shift work also includes activities that are related to electricity as an enabler, such as changes in the timing of daily activities, eating, sleeping, and social patterns. Other lifestyle activities may also differ in shift workers, such as physical activity and stress coping mechanisms, such as tobacco smoking and alcohol consumption; however, the directions of these confounding behaviors may be in opposite directions in different populations. Chronotype, the behavioral manifestation of underlying physiological phase in sleep-wake behavior (resulting in individual characteristics described as being a "morning lark" or "night owl"), which is partly a heritable trait, as well as other genetic traits, may also modify potential risks of adverse health outcomes in shift workers (Hansen and Lassen, 2012; Juda et al., 2013; Vetter et al., 2015). Furthermore, the role of the different exposures in shift workers may vary with the specific health outcome (e.g., eating patterns may be more important for obesity and metabolic disorders than for other health outcomes; co-exposures to carcinogens may affect cancer outcomes). The nature of the shift work itself may also be important in determining long-term health outcomes; for example, sedentary work may be associated with worse cardiovascular and metabolic outcomes than work involving significant levels of physical activity (De Rezende et al., 2014; Jermendy et al., 2012).

\subsection{Circadian disruption and adverse health effects}

Potential adverse health effects from light-induced circadian disruption are mediated in part by melatonin suppression. Light at night of sufficient level and duration, appropriate wavelength, and appropriate timing can shift the timing and/or reduce the amplitude of the nighttime melatonin signal, as may happen in night-shift workers (Dumont and Paquet, 2014). This may contribute to sleep changes and circadian disruption, which in turn affect a host of cellular mechanisms (such as metabolism and cell cycling) and neurobehavioral processes (such as mood regulation and cognitive outcomes). These disturbances may potentially lead to adverse health outcomes, as discussed below. Exposures to dim, warm-colored light during the day (e.g., $<50 \mathrm{~lx}$ at the eye of a $2700 \mathrm{~K}$ light source) may make matters worse. Studies have shown that higher levels of light during the daytime, including exposure to daylight, are associated with better sleep and mood in office workers (Boubekri et al., 2014; Figueiro et al., 2017).

The nighttime circadian melatonin signal in both rats and humans is known to exert an oncostatic role in a variety of cancers (Haus and Smolensky, 2013), most particularly in models of human breast cancer tumor growth (Blask et al., 2005; Blask et al., 2011). The nighttime melatonin signal modulates circadian rhythms in human breast cancer cell proliferation and metabolism and effects signal-transduction activity that restricts tumor growth. Exposure to dim LAN in rats and bright LAN in humans attenuates the melatonin signal and leads to the disruption of these circadian dynamics, resulting in tumor growth (Blask et al., 2005; Blask et al., 2014). In fact, the nighttime melatonin signal represents the only circadian-driven anticancer signal identified thus far in humans or in rats (Blask et al., 2005). Furthermore, suppression of the nocturnal circadian melatonin signal by LAN may lead not only to increased breast cancer risk but even to resistance of human breast cancer tissue to hormonal intervention and chemotherapy, as observed in preclinical human breast cancer studies (Dauchy et al., 2014; Xiang et al., 2015). The suppression of melatonin by LAN led to the hypothesis that LAN may play a role in the elevated incidence of breast cancer in some high-income countries; as increases in incidence of breast cancer have paralleled the expansion of LAN (Haus and Smolensky, 2013; Stevens, 1987; Stevens et al., 2014).

Aside from the direct effects of light on physiology, light also indirectly impacts daily rhythms by affecting hunger cues, acute behavior, and cognitive arousal levels (Chellappa et al., 2011). Thus, LAN may alter timing of food intake and the downstream effects of hormones and molecular signaling pathways (Fig. 1). Unnatural exposures to light, as either LAN or dim indoor lighting during the day, lead to changes in the expression of clock genes and proteins in the SCN, as well as melatonin and other hormones, which in turn lead to effects on sleep duration and quality and to disruption of central and peripheral circadian clocks. In addition, other activities, such as night-shift work, the timing of meals, and the composition of the foods eaten, act as entrainment or synchronizing cues for peripheral clocks, which also modify circadian rhythmicity.

Misalignment of sleep and circadian disruption have been linked to numerous adverse effects on performance, mental well-being, and physical health in animals or humans (e.g., Filipski et al., 2003; Grimaldi et al., 2016; Leproult et al., 2014). In the short term, these effects may include increased incidence of accidents and injuries, increased sick leave, mood disorders (including depression), weight gain, and social and family problems. As a consequence of chronic sleep disturbance and circadian disruption, adverse health outcomes may develop, including metabolic disorders and the related diseases of obesity and type 2 diabetes; cardiovascular-related diseases, including hypertension and ischemic stroke; neurological disorders; gastrointestinal ulcers; adverse reproductive outcomes; and various types of cancer. Moreover, circadian disruption may play a role in other unrecognized pathological mechanisms of human disease (Smolensky et al., 2016).

Irregular sleep-wake cycles may both result from and contribute to circadian disruption. The acute and transient symptoms, such as fatigue, sleepiness, indigestion, and irritability, of so-called "jet lag" that accompanies transmeridian travel are classic examples of this desynchronosis, or uncoupling of circadian rhythms. When travelers rapidly cross several time zones in one day, their 24-hour rhythms are suddenly desynchronized from the day-night cycle at their destination. Thus, biological rhythms such as sleeping and eating are wrongly timed, resulting in further misalignment of the master and peripheral clocks from the temporal features of the external environment and also from each other internally. Traveling eastward is more difficult than traveling westward and requires a longer period of adaptation for most people. Eastward travel requires a phase advance of the circadian pacemaker (i.e., an acute shortening of the 24-hour "day"). Following phase-advancing stimuli, some elements of the circadian system may adapt 
quickly but require several transient cycles in which remaining clock elements catch up (Illnerova et al., 1989; Reddy et al., 2002). Following a single advancing light pulse, shifts in behavior may not be completed for several cycles, whereas delaying shifts are fully manifest the following day. It is worth noting that for most travelers, the occurrence of jet lag is a transitory experience. Although acute transient circadian disruption typically is well tolerated, it can lead to negative health outcomes, such as exacerbating mood disorder in certain individuals, and chronic circadian disruption may potentially aggravate existing medical conditions whose etiopathology is circadian misalignment. One does not have to board a plane to experience the effects of circadian disruption. The vast majority of non-shift-working Americans wakes to the alarm clock during the work week and sleep in on the weekends, a phenomenon termed social jet lag. A large population study found the mean level of social jet lag is on the order of $90 \mathrm{~min}$ (Roenneberg et al., 2012). Social jet lag is associated with a set of symptoms that overlap with those of travel-associated jet lag, including increased sleepiness during waking times, decreased performance, and adverse effects on mood. When experienced chronically, social jetlag may lead to a suite of metabolic disorders known as "metabolic syndrome," which includes obesity, cardiovascular disease, and type 2 diabetes. In contrast to travel jet lag, social jet lag, whether associated with shift work or with "normal" life, is chronic in nature, and it may be the chronicity of circadian misalignment, rather than its absolute magnitude, that is important in shaping long-term health outcomes.

\subsubsection{Advancing the science for understanding potential health outcomes}

The Panel discussed scientific issues important for informing study designs and for advancing the understanding of health effects due to inappropriate exposures to light, including characterization of various exposures and end points (biomarkers and health outcomes). These issues are useful for evaluating and interpreting studies in the peer-reviewed literature, as well as for conducting new research.

\subsection{Characterization of light in human studies}

The Panel discussed several issues related to improving measurement of circadian-effective light (i.e., light that stimulates the circadian system) in human epidemiological and experimental studies. To date, characterization of indoor light exposures has been estimated from self-reports or retrospective questionnaires (e.g., bedroom brightness) in epidemiological studies of the relationship between exposure to electric light and cancer risks. Ecological studies of LAN in various geographical areas correlate light level, measured by satellite as a surrogate for exposure to LAN, with specific health outcomes. These studies, like all ecological studies, do not account for individual level exposures and co-exposures, and are therefore susceptible to confounding and misclassification bias.

A clear need exists for implementation of improved technologies to classify measures of actual circadian-effective light exposures experienced by individuals both indoors and outdoors during the daytime and at night. It is also important that researchers start reporting the light stimulus used in their studies in a more uniform manner, as proposed by Lucas et al. (2014). However, as noted by some Panel members, perfect exposure characterization may not be feasible in observational studies; therefore, exposure surrogates are needed to advance the understanding of causes of disease. In general, non-differential misclassification of exposure decreases the likelihood of detecting a true association in epidemiological studies (Blair et al., 2007).

Some members of the Panel presented data showing the limitations of using satellite images as surrogates for measuring circadian-effective light for individuals in ecological studies. Satellite data are filtered to more closely match the spectral sensitivity of two of the three cone photoreceptors (long- and medium-wavelength cones) that are responsible for visual acuity, which peaks close to $555 \mathrm{~nm}$, not the spectral sensitivity of the circadian system (as measured by melatonin suppression), which peaks close to $460 \mathrm{~nm}$ (Brainard et al., 2001; Thapan et al., 2001). Although these measures are imprecise, they may be able to separate individuals living in communities of high outdoor LAN exposure from those living in low LAN exposure (Stevens, 2011). However, it is unclear whether these ecological studies are able to separate the direct effects of light per se from the effects of light as an enabler of other behaviors potentially associated with poor health.

To advance exposure assessment used in epidemiology studies, the Panel suggested that field studies assess light exposure using calibrated devices, which can be used to validate detailed questionnaire data. Once validated, the questionnaires could be scaled up for use in large epidemiology studies and be designed to collect information on the type, duration, frequency, and timing of electric-light exposure and the timing of daylight exposure. These data could also be supplemented with data from new technologies, such as socially acceptable wearable monitoring devices that collect real-time data, and with data collection applications (apps) that allow users to enter information on eating and other behaviors.

More precise measurements of light exposure are required for experimental studies in humans to inform interventions to prevent acute melatonin suppression and circadian disruption. The Panel emphasized that it is important to improve the characterization of light that can lead to circadian disruption, including dose, light level, spectral composition (i.e., wavelength), distribution, timing, and duration to which an individual is exposed.

The action spectrum for melatonin suppression has been carefully measured for monochromatic light. Using these data, two spectral sensitivity functions and one mathematical model have been proposed and published in peer-reviewed journals, as described by Lucas et al. (2014). However, no spectral sensitivity function for characterizing light for the circadian system has been sanctioned by national or international standard-setting bodies. Nevertheless, using the Rea et al. (2005) mathematical model, one of the panel members generated and presented Fig. 3, which allows one to compare the relative effectiveness of light sources of various spectral power distributions and light levels for suppressing melatonin.

As shown in Fig. 3, for the same photopic light levels (lux) at the cornea, daylight is more effective at suppressing melatonin than is incandescent light, because daylight emits more energy in the short wavelength region (Rea et al., 2005; Rea et al., 2010). Night-time sensitivity to light (usually measured by melatonin suppression) is influenced by light exposure during the day, inter-individual sensitivity, chronotype, age, and photic history (Chang et al., 2011; Glickman et al., 2012; Hébert et al., 2002; Smith et al., 2004). It is also important to note in the discussion about health effects of LAN that occupational light exposures, such as those experienced by shift workers, are significantly higher than outdoor light exposures at night, and that most of the epidemiological work to date relating LAN to health risks has been limited to shift work.

Finally, the Panel suggested evaluating the interaction between experimentally induced diseases (e.g., using known toxicants) under different light conditions to determine how electric light may change susceptibility to environmental exposures. Evaluations in experimental animals could explore circadian chronotoxicities (differences in sensitivity to toxicants according to circadian time of exposure) under different light conditions. Observational epidemiological studies evaluating potential interactions between exposure to toxicants and LAN or among shift workers would also be informative.

\subsection{Characterization of exposures in studies of shift workers}

In most studies of shift workers, information on working arrangements is typically assessed from self-reported retrospective questionnaires or interviews, employment records, or job exposure matrices (e.g., individual occupational histories with survey data linking occupations to night work). Fully detailed classifications of shift work are rarely available, which include information about regular or irregular shift 


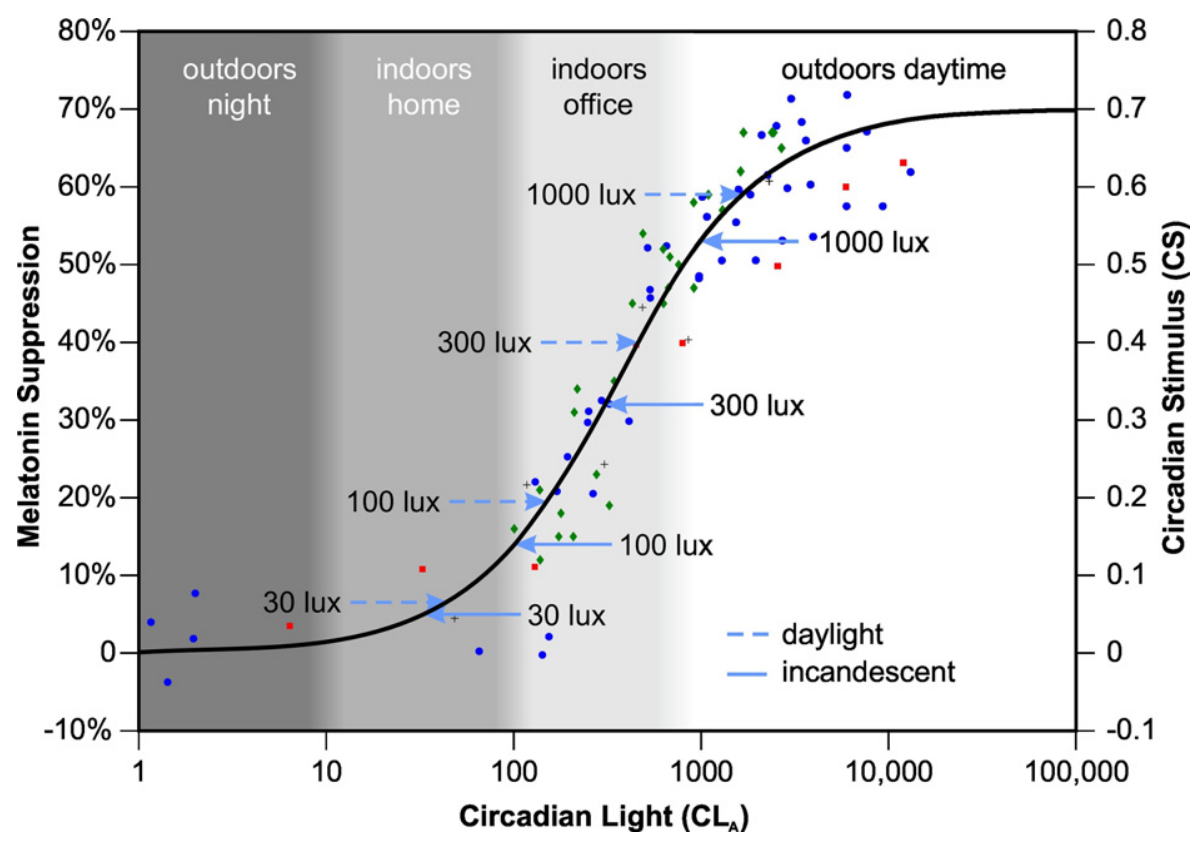

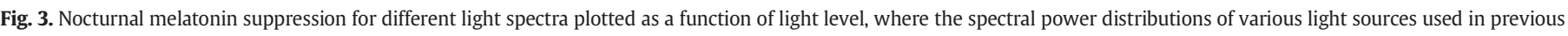

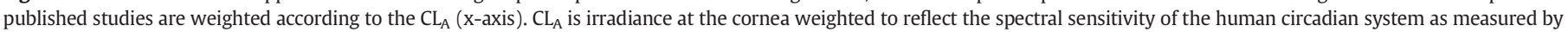

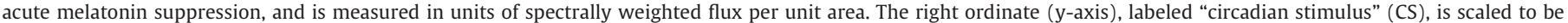

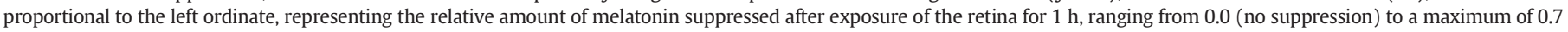
(70\% suppression).

schedules, time schedules of each shift, light level, permanent or rotating night shifts, direction and speed of rotation (e.g., intensity of shift work, consecutive night shifts, forward or backward rotations, permanent versus rotating shift work), and exposure window (e.g., age at first shift work or timing before or after full-term pregnancy). Rather, many studies have reported only years of working night shifts, without information on intensity (e.g., number and/or duration of night shifts per week and changes in shift schedule). Moreover, significant heterogeneity exists in definitions of "shift or night work" across the studies, which limits the utility of conducting a meta-analysis, as risk estimates from these studies cannot necessarily be combined. Furthermore, the nature of the light exposure of such employees during off-work hours is rarely assessed (Stevens et al., 2011).

In addition, co-exposures with shift work (e.g., known or presumed chemical risk factors for cancer and other health outcomes) may confound or modify the studied association. It has been suggested that shift workers and day workers may differ significantly in susceptibility to adverse effects from chemical exposures (Ward et al., 2010). Furthermore, risk factors for chronic diseases, such as poor diet, lack of physical activity, excess alcohol consumption and smoking, and being overweight, have frequently been reported among shift workers (Nea et al., 2015; Van Amelsvoort et al., 2006). Studies assessing the association between shift work and chronic health outcomes should obtain information on social patterns, lifestyle aspects (such as tobacco and alcohol use), and eating patterns (or body mass index), and evaluate their role as confounders, effect modifiers, or mediators of the association.

To date, only a few biomarkers, such as melatonin, cortisol, sex hormones, and epigenetic variables, have been studied in humans that could provide information on circadian disruption among shift workers. To be of maximal utility, most of these variables would require monitoring throughout an entire circadian cycle, and interpretation of the results for circadian disruption would require detailed understanding of how each may be acutely affected by environmental conditions. Detailed exposure assessment is needed to reduce measurement error and to suggest rational, empirically informed strategies for intervention. In addition, studies of shift workers are complicated because the exposure metrics that are related to adverse health effects have not been fully elucidated. In addition to collecting detailed information about the temporal patterning of the shifts worked, it is important to collect information on the different exposure components of shift work, including social patterns, lifestyle aspects (such as tobacco and alcohol use), eating patterns, light exposure (both at work and off work), sleeping patterns, and vigilance and cognition, all of which tend to be related (Fig. 4). New technologies, such as those mentioned above for light and the use of mobile phone apps and wearable activity trackers, could help facilitate exposure assessment of shift workers. In addition, it is important to collect information on potential confounders and effect modifiers, such as chronotype and other genetic traits.

The Nightingale study (Pijpe et al., 2014), a prospective cohort study on shift work and breast cancer among nurses in the Netherlands, is an example of a study currently in progress that has collected detailed information across all domains of the shift-work exposure scenario, including the shift-work system, cumulative exposure to shift work, and shift intensity. In addition to shift work, the questionnaire collects information on sociodemographics, reproductive history, education, other occupational exposure, mobile phone use, residential history, current sleeping habits (including disruption of sleep not related to occupational shift work), and medical and family history of diseases. To more fully characterize the complex exposure scenario of shift work, a substudy of the Nightingale study called "Klokwerk" is collecting information on various exposures through questionnaire data, 24-hour recall logs, employer data, light sensors, and biomarkers in the blood, urine, and feces. Another example of a nationwide prospective cohort is the Danish Working Hours Database, which, on an individual level, covers exact daily working time from payrolls for all employees in all hospitals (Garde et al., 2016). The unique personal number applied to all residents in Denmark makes it possible to link this information with information from health registries, such as the national cancer registry or the national hospital register, for research purposes.

\subsection{Animal models of LAN and shift work}

The Panel noted that although animal models of shift work and exposures to LAN (primarily rats and mice) do not fully replicate complex, overlapping exposure scenarios in humans, they do play a key role in 


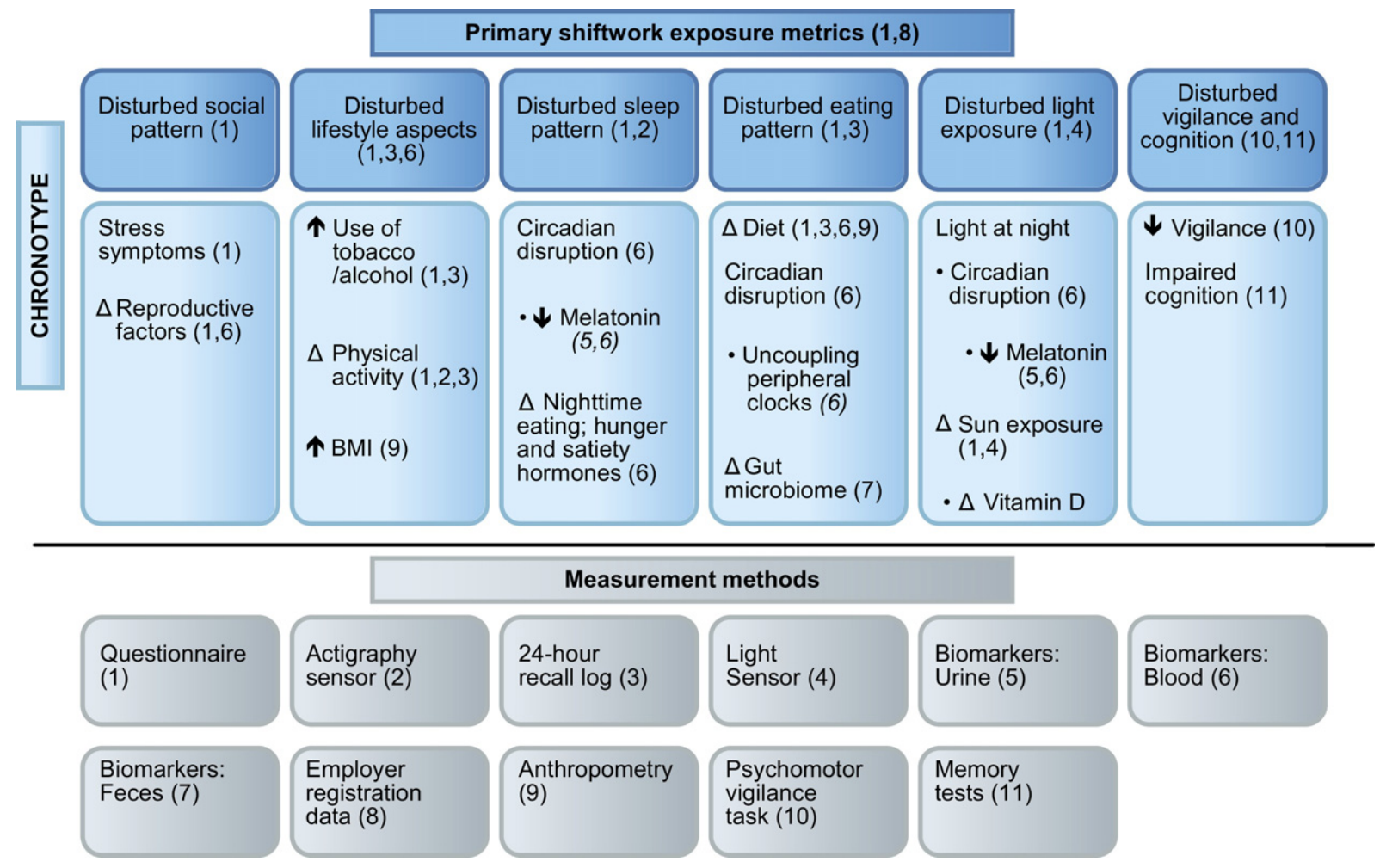

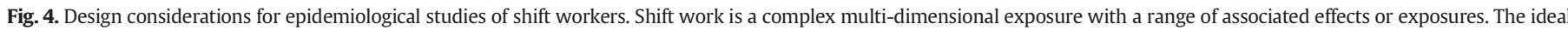

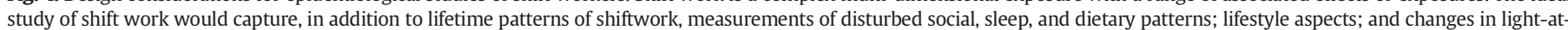

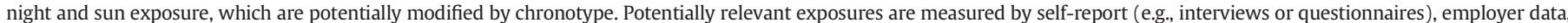

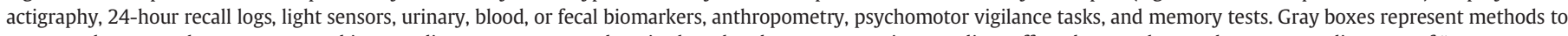

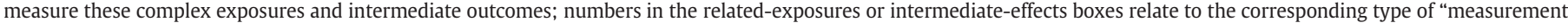
methods" to assess the factors; ( $\Delta=$ change, arrows indicate direction of change).

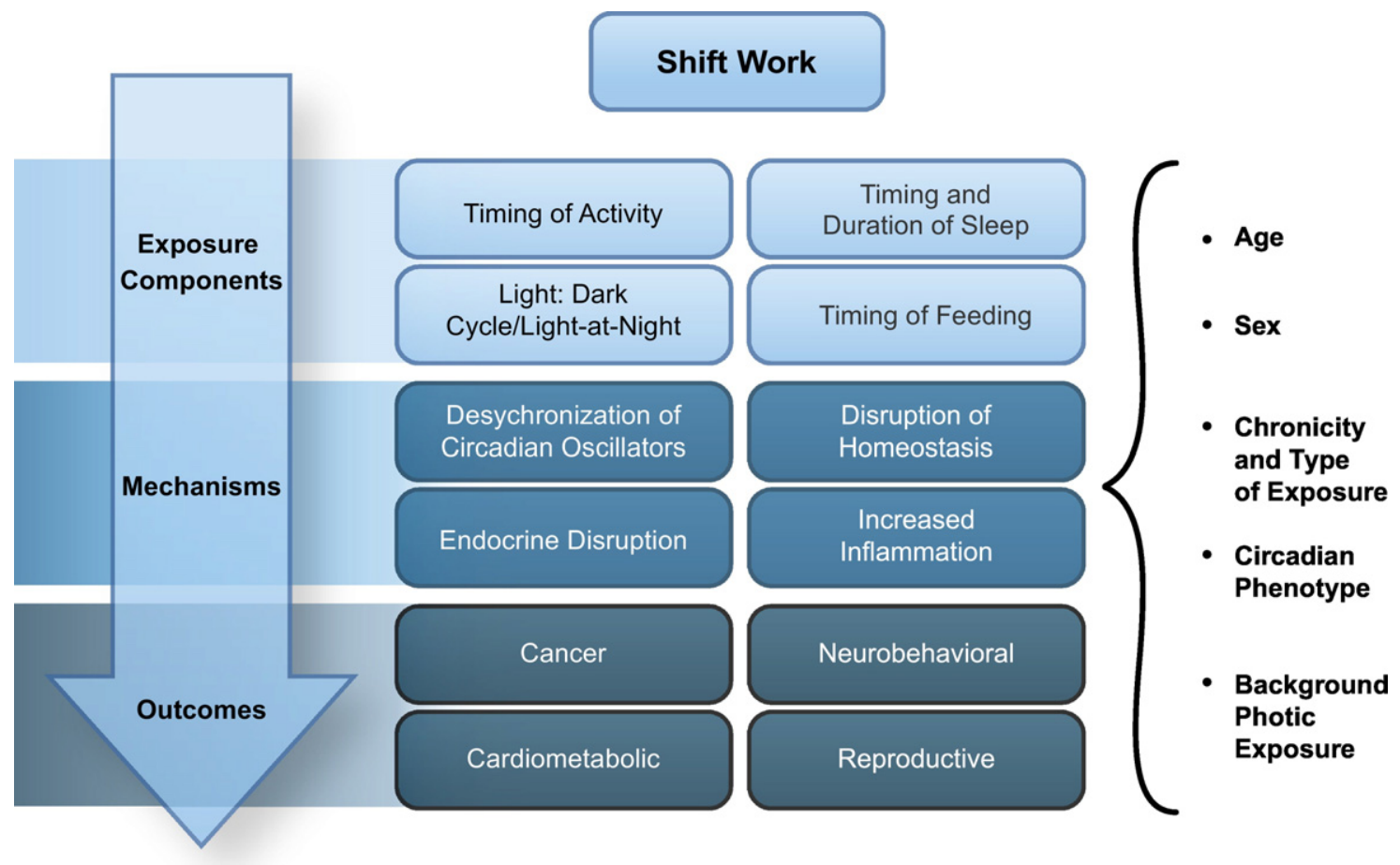

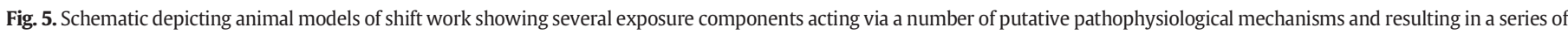

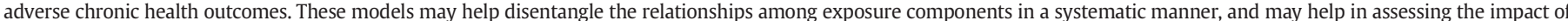

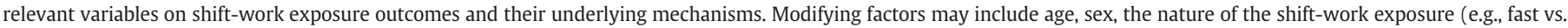

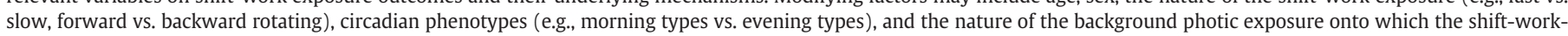
related light-dark cycle is imposed (e.g., long, summer-like photoperiods vs. short, winter-like photoperiods). 
understanding specific exposures and mechanisms, and provide evidence for interventions. These animal studies have either evaluated the direct effects of light or have tried to model shift work by exposing animals to repeated phase shifts in the light-dark cycle, and have investigated a variety of end points and health outcomes, including cancer; cardiovascular disease; mood and behavior disorders; effects on the immune, neurological, or reproductive systems; energy metabolism and obesity; and changes in the structure of gut microbial communities (Summa and Turek, 2014; Voigt et al., 2014) (Fig. 5). Animal studies will also be crucial in testing hypotheses related to interventions, such as the effect of meal timing or meal composition on potentially alleviating some of the adverse effects due to circadian disruption. Animal models may offer the advantage of allowing relatively easy assessment of the importance of chronicity of exposure on health-related biomarkers of circadian disruption, including the development of timeof-day-independent biomarkers that may be used in human studies, such as a set of liver-transcriptome-based biomarkers recently reported by Van Dycke et al. (2015).

Studies to evaluate effects of light as a direct effector have primarily altered the light timing or level and have usually exposed animals to continuous bright light, constant dim light in the dark phase, or intermittent light exposure in the dark phase (e.g., 1-minute exposure to light every few hours) or shortened or lengthened the photoperiods of light and dark, with 12-hour light-dark days for the control groups. The Panel suggested that animal facilities should be constructed with light-tight rooms for housing animals in the dark phase and that room lighting specifications should be standardized across all animal studies. They also noted that while animal studies are informative, nocturnal animals are more sensitive to light than humans, and it is therefore important to translate metrics from animals to humans appropriately. Dim light in animal studies reported in the peer-reviewed literature typically ranged from 0.21 to $5 \mathrm{~lx}$, with the latter corresponding to light intensity between a full moon $(<1 \mathrm{~lx})$ and twilight $(\sim 10 \mathrm{~lx})$.

The Panel suggested studies on dim LAN at lower light levels than commonly reported in the literature and the use of diurnal models with both male and female animals to more closely replicate human exposures and effects. As a short-term goal, the Panel suggested conducting studies in experimental animals using study designs similar to those used by Nelson and colleagues, which used lower, more biologically relevant doses of light to better characterize the dose-response relationship. These studies compared health-related outcomes in animals (mice, rats, or hamsters) exposed to $16 \mathrm{~h}$ of bright broad-spectrum (white) light (150 lx) and $8 \mathrm{~h}$ of dim light (5 lx) with outcomes in control animals exposed to $16 \mathrm{~h}$ of bright light ( $150 \mathrm{~lx}$ ) and $8 \mathrm{~h}$ of complete darkness (e.g., Aubrecht et al., 2014; Aubrecht et al., 2015; Bedrosian et al., 2011; Bedrosian et al., 2013a; Fonken et al., 2012).

The Panel also noted that some strains of laboratory mice either (1) do not produce melatonin even though they have melatonin receptors or (2) have abnormal patterns of melatonin production (e.g., a nocturnal spike or production during the light phase). These species differences between humans and animals should be considered in both the interpretation of studies in the peer-reviewed literature and in the design of new studies in mice. The recent discovery that melatonin can adversely affect glucose-induced insulin release from the pancreas raises a mechanistic link between melatonin and metabolism (Tuomi et al., 2016). In light of this finding, controlled animal experiments using strains that have intact melatonin production and receptors combined with controlled lighting and diet will be important for future research. The Panel also noted a need for the use of a wider range of animal models in the study of LAN and shift work, in order to address the nature of species-specific differences that may be informative for human studies.

\subsection{Biomarkers of circadian disruption and adverse outcomes}

The classic biomarkers of circadian disruption in humans are core body temperature, the sleep-wake cycle, and circulating melatonin and its main metabolite 6-sulfatoxymelatonin, measured most frequently in urine, but also in saliva and blood (Mirick and Davis, 2008). Less frequently, other circulating hormones, such as cortisol and sex hormones, have also been measured. Studies using animal models of shift work or animals exposed to various light regimes most often use the rhythm of locomotor activity and melatonin (in the light studies) as biomarkers of circadian disruption. Other measures of circadian disruption in animal models include body temperature, corticosterone concentration, and expression of circadian-related and clock-related genes.

Because the levels of such circadian biomarkers fluctuate with time of day, sampling at multiple time points is likely to be necessary, especially among shift workers and others who may or may not be circadian-synchronized. In the past, sampling of melatonin has varied across studies, with some collecting 24-hour specimens and others collecting the first morning void. The latter may fairly represent the nightly production of night-sleeping people, but may not be a fair measure for night-working people. The identification of biomarkers that are independent of circadian time per se would be useful for large populationbased epidemiological studies. To this end, the Panel referred the NTP to recent work reporting universal biomarkers of circadian disruption that are independent of time sampling, which identified 15 genes with increased expression in mouse liver after simulated chronic jet lag (Van Dycke et al., 2015). An increased level of the protein encoded by one of these genes, CD36, was also observed in the serum of jet-lagged animals, indicating the potential utility of this marker as a non-invasive biomarker of circadian disruption in human studies.

Other emerging measures of circadian disruption that require more investigation include measurement of elevated cytokine expression and other markers of inflammation using commercially available kits; epigenetic effects, such as promoter methylation; effects on the microbiome and resultant health outcomes; genomic characterization of chronotype or diurnal preference; and genetic polymorphisms that affect individual differences in the sensitivity to circadian effects and health risks. An important observation made by the Panel was that few to no studies have adequately characterized the degree of circadian disruption in conjunction with the measurement of specific health outcomes in humans (Roenneberg and Merrow, 2016). The Panel also noted the need to use multiple biomarkers for circadian disruption in order to appreciate the desynchronization of individual circadian rhythms (e.g., desynchronization of the hepatic clock from the SCN clock or of melatonin rhythm from sleep timing). The Panel noted the need for specific biomarkers of circadian disruption that are relevant for understanding the mechanisms and risk for developing specific negative outcomes.

\subsubsection{Protecting public health}

The impact on public health from either direct or indirect effects of electric lighting is of utmost concern. Unnatural light exposures (LAN and/or dim light during the day) and shift work are very common and in some cases unavoidable components of our society. In addition, the potential adverse health outcomes - such as cancers, cardiovascular disease, obesity, adverse pregnancy outcomes, and metabolic diseases - are common. Since the March 2016 workshop, the French Agency for Food, Environmental and Occupational Health \& Safety released its assessment of the health risks associated with night work (ANSES, 2016). It concluded that working at night was associated with "proven" effects for sleep disturbances (sleep quality, sleep time, and sleepiness and alertness) and metabolic syndrome, and "probable" effects for cognitive performances, cardiovascular diseases, metabolic-related disorders (obesity or overweight and type 2 diabetes), and breast cancer and other types of cancer (prostate, ovary, pancreas, and colorectal). As people will likely continue to work alternative shift schedules, and society will need to use electric lighting, the key question to ask is, what reasonable changes can people make to decrease their risk of potential adverse health effects? 
Although night-shift work presents a complex exposure scenario, where electric light can be both an effector and an enabler, interventions that minimize the effects of light and shift work on circadian disruption may help prevent adverse health outcomes. Ideally, interventions could be implemented now, rather than after waiting until studies evaluating the mechanisms of disease and intervention are completed. To date, several experimental studies of sleep in humans have evaluated the effects of lighting characteristics - such as level, duration, wavelength, and biological timing - on biomarkers of circadian disruption, such as melatonin, cortisol, and body temperature. For additional information, see workshop background materials available at http://ntp.niehs.nih.gov/go/workshop_ALAN. Some studies have also evaluated whether interventions such as using blue-light-blocking goggles affect circadian biology. Other studies suggest that using light sources with low emission at short wavelengths minimizes melatonin suppression without greatly affecting performance and alertness at night (Kayumov et al., 2005; Rahman et al., 2017; Van de Werken et al., 2013).

The American Medical Association recently advised minimizing and controlling short-wavelength (blue) and light-emitting-diode lighting because of health and safety concerns (http://www.ama-assn.org/ ama/ama-wire/post/physicians-timely-public-health-issues). It should be noted, however, that levels of outdoor LAN exposures at night are much lower (recommended average horizontal illuminance is 18 photopic lux (Illuminating Engineering Society, 2014), which is approximately 6 photopic lux at eye level) than light levels experienced by shift workers working at night (between 50 and 100 photopic lux at the eye, with some exposures exceeding $200 \mathrm{~lx}$ (Hunter and Figueiro, 2017)).

The Panel noted that industry is becoming aware of the potential adverse effects of short-wavelength light at night, as it provides electronicdevice users the opportunity to reduce short-wavelength light emissions from the screen. Nonetheless, recommendations focused only on reducing exposure to short-wavelength light may not prevent adverse health outcomes, because other lighting characteristics, such as the amount and duration of exposure, play a role in how LAN affects nocturnal melatonin production and broader circadian function. One potential future strategy for mitigating against cancer risk or treatment failures may be the use of "near"-physiological melatonin supplementation or replacement, especially in high-risk groups for which the type of circadian disruption involved is restricted to nighttime melatonin suppression. Other potential interventions may focus on meal timing and diet.

Studies in experimental animals have also investigated interventions in models of depression and stroke damage after exposure to LAN, such as using red light instead of blue light, to minimize adverse health outcomes. For example, a Panel member presented data from a study that found less depression-like behavior and fewer alterations in neuronal structure in hamsters exposed to dim red light during the dark phase than in hamsters exposed to dim white or dim blue light during the dark phase (Bedrosian et al., 2013b). The Panel's advice on improving exposure and outcome assessments and studying mechanisms will help inform intervention strategies.

\section{Conclusions}

An important outcome of the workshop was to increase the visibility of these pervasive and often unavoidable exposures and their associations with potential adverse health effects. Same-day attendees, either in person or via the webinar, exceeded 150 people from diverse organizations, including government, industry, academia, and non-profits, along with other concerned citizens. Videos of the workshop and summary materials remain available on the NTP website (http://ntp.niehs. nih.gov/go/workshop_ALAN).

The workshop deliberations provided input on approaches for conducting health hazard evaluations, such as guidance on problem formulation, protocol development (e.g., types of studies to include and
Box 1

Key issues for evaluating health effects associated with electric light.

- Many characteristics of electric light exposure are related to circadian disruption, including level, spectrum, distribution, timing, duration, and photic history. In addition, light exposure during the day influences nighttime light sensitivity, so attention to the daily pattern of light exposure is important for maintaining health.

- In addition to direct effects of unnatural light at night (LAN), LAN enables activities that allow individuals to eat, sleep, and conduct other activities 24/7, which is associated with circadian disruption and adverse health effects.

- Shift work is a complex exposure scenario, involving both exposure to light and activities enabled by light.

- LAN may have a significant public health impact due to the pervasive exposure and its potential to increase the risk of common diseases, such as cancer, obesity, and heart disease.

- Understanding potential mechanisms and characteristics of light or shift work that are related to circadian disruption or biomarkers of disease may help identify interventions to protect public health.

exclude), interpretation of data from various animal models, and factors to consider when trying to synthesize across different bodies of evidence (see Boxes 1 and 2 for key issues). NTP will use this input in its

Box 2

Research needs for the field.

- Conduct field studies on light exposures using calibrated devices, mobile applications, and detailed questionnaire data that can be scaled up for use in large epidemiology studies.

- Collect information (e.g., questionnaires, biomarkers) that provides comprehensive characterization of shift work (e.g., scheduling and nature) and other "exposures," such as light exposure and sleep and eating patterns.

- Develop and test interventions for shift workers that minimize the effects of light and shift work on circadian disruption and thereby help prevent adverse safety and health outcomes.

- Develop non-circadian markers of circadian disruption that could be employed in epidemiological and intervention studies.

- Measure biomarkers at multiple time points, to determine optimal times for larger-scale studies.

- Conduct studies in animals to model relevant components of shift-work exposure on biomarkers of circadian function and health outcomes.

- Conduct studies in animals that involve both acute and chronic repeated phase shifts in the light-dark cycle, exposure to constant light, feeding at the "wrong" time of day, and acutely or chronically shortened sleep. Such studies have the potential to elucidate mechanisms by which disrupted sleep or circadian rhythms lead to adverse health outcomes, and for the exploitation of these models for testing therapeutic interventions (Fig. 5).

- Conduct studies in animals with dim LAN at lower light levels, and use diurnal models to more closely replicate human exposures and effects.

- Evaluate interactions between chemical and light exposures in humans and animals. 
cancer hazard evaluation for review of night shift work and electriclight exposures for the RoC.

Delineating research gaps and study-design considerations will help ensure that future studies more comprehensively characterize and measure the relevant light-related exposures and link these exposures to additional and time-independent biomarkers of circadian disruption and to biomarkers of adverse health outcomes (see Box 2). In this way, existing information on interventions can be supplemented with additional information, leading to realistic, implementable, and effective intervention strategies to minimize the occurrence of adverse health outcomes due to these exposures.

\section{Funding}

This research did not receive any specific grants from funding agencies in the public, commercial, or not-for-profit sectors.

\section{Disclaimer}

The findings and conclusions in this manuscript are those of the author(s) and do not necessarily represent the views of the National Toxicology Program or the National Institute for Occupational Safety and Health.

\section{Acknowledgments}

The authors would like to acknowledge Dr. Gloria Jahnke, Dr. Katie Pelch, Dr. Kyla Taylor, Ms. Whitney Mitchell, and Ms. Courtney Skuce for their support at the workshop or for preparation of background materials for the workshop.

\section{References}

ANSES, 2016. French agency for food, environmental and occupational health \& safety Available. https://www.anses.fr/en.

Aubrecht, T.G., Weil, Z.M., Nelson, R.J., 2014. Dim light at night interferes with the development of the short-day phenotype and impairs cell-mediated immunity in Siberian hamsters (Phodopus sungorus). J. Exp. Zool. A Ecol. Genet. Physiol. 321, 450-456.

Aubrecht, T.G., Jenkins, R., Nelson, R.J., 2015. Dim light at night increases body mass of female mice. Chronobiol. Int. 32, 557-560.

Bedrosian, T.A., Fonken, L.K., Walton, J.C., Nelson, R.J., 2011. Chronic exposure to dim light at night suppresses immune responses in Siberian hamsters. Biol. Lett. 7, 468-471.

Bedrosian, T.A., Aubrecht, T.G., Kaugars, K.E., Weil, Z.M., Nelson, R.J., 2013a. Artificial light at night alters delayed-type hypersensitivity reaction in response to acute stress in $\mathrm{Si}$ berian hamsters. Brain Behav. Immun. 34, 39-42.

Bedrosian, T.A., Vaughn, C.A., Galan, A., Daye, G., Weil, Z.M., Nelson, R.J., 2013b. Nocturnal light exposure impairs affective responses in a wavelength-dependent manner. J. Neurosci. 33, 13081-13087.

Blair, A., Stewart, P., Lubin, J.H., Forastiere, F., 2007. Methodological issues regarding confounding and exposure misclassification in epidemiological studies of occupationa exposures. Am. J. Ind. Med. 50, 199-207.

Blask, D.E., Brainard, G.C., Dauchy, R.T., Hanifin, J.P., Davidson, L.K., Krause, J.A., et al., 2005. Melatonin-depleted blood from premenopausal women exposed to light at night stimulates growth of human breast cancer xenografts in nude rats. Cancer Res. 65, 11174-11184.

Blask, D.E., Dauchy, R.T., Brainard, G.C., Hanifin, J.P., 2009. Circadian stage-dependent inhibition of human breast cancer metabolism and growth by the nocturnal melatonin signal: consequences of its disruption by light at night in rats and women. Integr. Cancer Ther. 8, 347-353.

Blask, D.E., Hill, S.M., Dauchy, R.T., Xiang, S., Yuan, L., Duplessis, T., et al., 2011. Circadian regulation of molecular, dietary, and metabolic signaling mechanisms of human breast cancer growth by the nocturnal melatonin signal and the consequences of its disruption by light at night. J. Pineal Res. 51, 259-269.

Blask, D.E., Dauchy, R.T., Dauchy, E.M., Mao, L., Hill, S.M., Greene, M.W., et al., 2014. Light exposure at night disrupts host/cancer circadian regulatory dynamics: impact on the Warburg effect, lipid signaling and tumor growth prevention. PLoS One 9, e102776.

Boubekri, M., Cheung, I.N., Reid, K.J., Wang, C.H., Zee, P.C., 2014. Impact of windows and daylight exposure on overall health and sleep quality of office workers: a case-control pilot study. J. Clin. Sleep Med. 10, 603-611.

Brainard, G.C., Hanifin, J.P., Greeson, J.M., Byrne, B., Glickman, G., Gerner, E., et al., 2001. Action spectrum for melatonin regulation in humans: evidence for a novel circadian photoreceptor. J. Neurosci. 21, 6405-6412.

Chang, A.M., Scheer, F.A., Czeisler, C.A., 2011. The human circadian system adapts to prior photic history. J. Physiol. 589, 1095-1102.

Chellappa, S.L., Gordijn, M.C., Cajochen, C., 2011. Can light make us bright? Effects of light on cognition and sleep. Prog. Brain Res. 190, 119-133.
Crowley, S.J., Carskadon, M.A., 2010. Modifications to weekend recovery sleep delay circadian phase in older adolescents. Chronobiol. Int. 27, 1469-1492.

Dauchy, R.T., Xiang, S., Mao, L., Brimer, S., Wren, M.A., Yuan, L., et al., 2014. Circadian and melatonin disruption by exposure to light at night drives intrinsic resistance to tamoxifen therapy in breast cancer. Cancer Res. 74, 4099-4110.

De Rezende, L.F., Rodrigues Lopes, M., Rey-Lopez, J.P., Matsudo, V.K., Luiz, Odo C., 2014. Sedentary behavior and health outcomes: an overview of systematic reviews. PLoS One 9, e105620.

Dumont, M., Paquet, J., 2014. Progressive decrease of melatonin production over consecutive days of simulated night work. Chronobiol. Int. 31, 1231-1238.

Falchi, F., Cinzano, P., Duriscoe, D., Kyba, C.C., Elvidge, C.D., Baugh, K., et al., 2016. The new world atlas of artificial night sky brightness. Sci. Adv. 2, e1600377.

Figueiro, M., Overington, D., 2015. Self-luminous devices and melatonin suppression in adolescents. Light. Res. Technol. 48, 966-975.

Figueiro, M.G., Steverson, B., Heerwagen, J., Kampschroer, K., Hunter, C.M., Gonzales, K., et al., 2017. The impact of daytime light exposures on sleep and mood in office workers. Sleep Health 3, 204-215.

Filipski, E., King, V.M., Li, X., Granda, T.G., Mormont, M.C., Claustrat, B., et al., 2003. Disruption of circadian coordination accelerates malignant growth in mice. Pathol. Biol. 51, 216-219.

Fonken, L.K., Kitsmiller, E., Smale, L., Nelson, R.J., 2012. Dim nighttime light impairs cognition and provokes depressive-like responses in a diurnal rodent. J. Biol. Rhythm. 27, 319-327.

Garde, A.H., Hansen, J., Kolstad, H.A., Larsen, A.D., Hansen, A.M., 2016. How do different definitions of night shift affect the exposure assessment of night work? Chronobiol. Int. 33, 595-598.

Glickman, G., Webb, I.C., Elliott, J.A., Baltazar, R.M., Reale, M.E., Lehman, M.N., et al., 2012. Photic sensitivity for circadian response to light varies with photoperiod. J. Biol. Rhythm. 27, 308-318.

Grimaldi, D., Carter, J.R., Van Cauter, E., Leproult, R., 2016. Adverse impact of sleep restriction and circadian misalignment on autonomic function in healthy young adults. Hypertension $68,243-250$.

Hansen, J., Lassen, C.F., 2012. Nested case-control study of night shift work and breast cancer risk among women in the Danish military. Occup. Environ. Med. 69, 551-556.

Haus, E.L., Smolensky, M.H., 2013. Shift work and cancer risk: potential mechanistic roles of circadian disruption, light at night, and sleep deprivation. Sleep Med. Rev. 17, 273-284

Hébert, M., Martin, S.K., Lee, C., Eastman, C.I., 2002. The effects of prior light history on the suppression of melatonin by light in humans. J. Pineal Res. 33, 198-203.

Hunter, C.M., Figueiro, M.G., 2017. Measuring light at night and melatonin levels in shift workers: a review of the literature. Biol. Res. Nurs. 19, 365-374.

IARC, 2010. Shift work. Painting, Firefighting, and Shiftwork. vol. 98. International Agency for Research on Cancer, Lyon, France, pp. 563-764.

Illnerova, H., Vanecek, J., Hoffmann, K., 1989. Different mechanisms of phase delays and phase advances of the circadian rhythm in rat pineal $\mathrm{N}$-acetyltransferase activity. J. Biol. Rhythm. 4, 187-200.

Jermendy, G., Nadas, J., Hegyi, I., Vasas, I., Hidvegi, T., 2012. Assessment of cardiometabolic risk among shift workers in Hungary. Health Qual. Life Outcomes 10, 18.

Juda, M., Vetter, C., Roenneberg, T., 2013. Chronotype modulates sleep duration, sleep quality, and social jet lag in shift-workers. J. Biol. Rhythm. 28, 141-151.

Kandalepas, P.C., Mitchell, J.W., Gillette, M.U., 2016. Melatonin signal transduction pathways require E-box-mediated transcription of Per1 and er2 to reset the SCN clock at dusk. PLoS One 11, e0157824.

Kayumov, L., Casper, R.F., Hawa, R.J., Perelman, B., Chung, S.A., Sokalsky, S., et al., 2005. Blocking low-wavelength light prevents nocturnal melatonin suppression with no adverse effect on performance during simulated shift work. J. Clin. Endocrinol. Metab. 90, 2755-2761.

Leproult, R., Holmback, U., Van Cauter, E., 2014. Circadian misalignment augments markers of insulin resistance and inflammation, independently of sleep loss. Diabetes 63, 1860-1869.

Lucas, R.J., Peirson, S.N., Berson, D.M., Brown, T.M., Cooper, H.M., Czeisler, C.A., et al., 2014. Measuring and using light in the melanopsin age. Trends Neurosci. 37, 1-9.

Mirick, D.K., Davis, S., 2008. Melatonin as a biomarker of circadian dysregulation. Cancer Epidemiol. Biomark. Prev. 17, 3306-3313.

Nea, F.M., Kearney, J., Livingstone, M.B., Pourshahidi, L.K., Corish, C.A., 2015. Dietary and lifestyle habits and the associated health risks in shift workers. Nutr. Res. Rev. 28, 143-166.

Ohayon, M.M., Milesi, C., 2016. Artificial outdoor nighttime lights associate with altered sleep behavior in the american general population. Sleep 39, 1311-1320.

Pijpe, A., Slottje, P., van Pelt, C., Stehmann, F., Kromhout, H., van Leeuwen, F.E., et al., 2014. The nightingale study: rationale, study design and baseline characteristics of a prospective cohort study on shift work and breast cancer risk among nurses. BMC Cancer $14,47$.

Rahman, S.A., St Hilaire, M.A., Lockley, S.W., 2017. The effects of spectral tuning of evening ambient light on melatonin suppression, alertness and sleep. Physiol. Behav. 177, 221-229.

Rea, M.S., Figueiro, M.G., Bullough, J.D., Bierman, A., 2005. A model of phototransduction by the human circadian system. Brain Res. Brain Res. Rev. 50, 213-228.

Rea, M.S., Figueiro, M.G., Bierman, A., Bullough, J.D., 2010. Circadian light. J. Circadian Rhythms 8, 2 .

Reddy, A.B., Field, M.D., Maywood, E.S., Hastings, M.H., 2002. Differential resynchronisation of circadian clock gene expression within the suprachiasmatic nuclei of mice subjected to experimental jet lag. J. Neurosci. 22, 7326-7330.

Roenneberg, T., Merrow, M., 2016. The circadian clock and human health. Curr. Biol. 26, R432-R443.

Roenneberg, T., Allebrandt, K.V., Merrow, M., Vetter, C., 2012. Social jetlag and obesity. Curr. Biol. 22, 939-943. 
Smith, K.A., Schoen, M.W., Czeisler, C.A., 2004. Adaptation of human pineal melatonin suppression by recent photic history. J. Clin. Endocrinol. Metab. 89, 3610-3614.

Smolensky, M.H., Hermida, R.C., Reinberg, A., Sackett-Lundeen, L., Portaluppi, F., 2016. Circadian disruption: new clinical perspective of disease pathology and basis for chronotherapeutic intervention. Chronobiol. Int. 1-19.

Society, Illuminating Engineering, 2014. Roadway Lighting. ANSI/IES RP-8-14. Illuminating Engineering Society, New York, NY.

Stevens, R.G., 1987. Electric power use and breast cancer: a hypothesis. Am. J. Epidemiol. 125, 556-561.

Stevens, R.G., 2011. Testing the light-at-night (LAN) theory for breast cancer causation. Chronobiol. Int. 28, 653-656.

Stevens, R.G., Hansen, J., Costa, G., Haus, E., Kauppinen, T., Aronson, K.J., et al., 2011. Considerations of circadian impact for defining "shift work" in cancer studies: IARC working group report. Occup. Environ. Med. 68, 154-162.

Stevens, R.G., Brainard, G.C., Blask, D.E., Lockley, S.W., Motta, M.E., 2014. Breast cancer and circadian disruption from electric lighting in the modern world. CA Cancer J. Clin. 64, 207-218.

Straif, K., Baan, R., Grosse, Y., Secretan, B., El Ghissassi, F., Bouvard, V., et al., 2007. Carcinogenicity of shift-work, painting, and fire-fighting. Lancet Oncol. 8, 1065-1066.

Summa, K.C., Turek, F.W., 2014. Chapter 4. Biomedical effects of circadian rhythm disturbances. In: Redline, S., Berger, N.A. (Eds.), Impact of Sleep and Sleep Disturbances on Obesity and Cancer. Springer-Verlag, New York, pp. 77-99.

Thapan, K., Arendt, J., Skene, D.J., 2001. An action spectrum for melatonin suppression: evidence for a novel non-rod, non-cone photoreceptor system in humans. J. Physiol. $535,261-267$.
Tuomi, T., Nagorny, C.L., Singh, P., Bennet, H., Yu, Q., Alenkvist, I., et al., 2016. Increased melatonin signaling is a risk factor for type 2 diabetes. Cell Metab. 23, 1067-1077.

Van Amelsvoort, L.G., Jansen, N.W., Kant, I., 2006. Smoking among shift workers: more than a confounding factor. Chronobiol. Int. 23, 1105-1113.

Van de Werken, M., Gimenez, M.C., de Vries, B., Beersma, D.G., Gordijn, M.C., 2013. Shortwavelength attenuated polychromatic white light during work at night: limited melatonin suppression without substantial decline of alertness. Chronobiol. Int. 30 843-854.

Van Dycke, K.C., Pennings, J.L., van Oostrom, C.T., van Kerkhof, L.W., van Steeg, H., van der Horst, G.T., et al., 2015. Biomarkers for circadian rhythm disruption independent of time of day. PLoS One 10, e0127075.

Vetter, C., Fischer, D., Matera, J.L., Roenneberg, T., 2015. Aligning work and circadian time in shift workers improves sleep and reduces circadian disruption. Curr. Biol. 25, 907-911.

Voigt, R.M., Forsyth, C.B., Green, S.J., Mutlu, E., Engen, P., Vitaterna, M.H., et al., 2014. Circadian disorganization alters intestinal microbiota. PLoS One 9, e97500.

Ward, E.M., Schulte, P.A., Straif, K., Hopf, N.B., Caldwell, J.C., Carreon, T., et al., 2010. Research recommendations for selected iarc-classified agents. Environ. Health Perspect. 118, 1355-1362.

Xiang, S., Dauchy, R.T., Hauch, A., Mao, L., Yuan, L., Wren, M.A., et al., 2015. Doxorubicin resistance in breast cancer is driven by light at night-induced disruption of the circadian melatonin signal. J. Pineal Res. 59, 60-69. 